\title{
Gaussian approximation of perturbed chi-square risks
}

\author{
Krzysztof DęBicki ${ }^{*, \dagger}$, Enkelejd Hashorva ${ }^{\dagger}$, And Lanpeng Ji ${ }^{\dagger}$,
}

In this paper we show that the conditional distribution of perturbed chi-square risks can be approximated by certain distributions including the Gaussian distributions. Our results are of interest for conditional extreme value models and multivariate extremes as shown in three applications.

AMS 2000 SUBJECT ClASSIFICATIONS: Primary 60G15; secondary $60 \mathrm{G} 70$.

Keywords AND Phrases: Gaussian approximation, Chisquare distribution, Berman's sojourn limit theorem, Conditional limit law, Hüsler-Reiss distribution.

\section{INTRODUCTION}

Let $\left(X_{i 1}, X_{i 2}\right), i \geq 1$ be independent bivariate Gaussian random vectors with $N(0,1)$ distributed marginals and correlation coefficient $\rho \in(-1,1)$. We have the following stochastic representation

$$
\left(X_{i 1}, X_{i 2}\right) \stackrel{d}{=}\left(X_{i 1}, \rho X_{i 1}+\sqrt{1-\rho^{2}} W_{i}\right), \quad i \geq 1,
$$

with $W_{i}, i \geq 1$ being independent $N(0,1)$ random variables (rvs) which are further independent of $X_{i 1}, i \geq 1$. For fixed $m \geq 2$ we define a bivariate chi-square random vector $\left(\zeta_{1}, \zeta_{2}\right)$ by

$$
\zeta_{1}=\sum_{i=1}^{m} X_{i 1}^{2}, \quad \zeta_{2}=\sum_{i=1}^{m} X_{i 2}^{2}
$$

Apart from the case $\rho=0$, the bivariate random vector $\left(\zeta_{1}, \zeta_{2}\right)$ has dependent components. By a direct analytic proof (see Appendix) it follows that, as $v \rightarrow \infty$, the conditional risk (defined almost surely)

$$
\zeta_{v}^{*}:=\frac{\zeta_{2}-\rho^{2} v}{2 \rho \sqrt{\left(1-\rho^{2}\right) v}} \mid\left(\zeta_{1}=v\right)
$$

arXiv: 1309.4975

*Partial support by NCN Grant No 2011/01/B/ST1/01521 (20112013).

${ }^{\dagger}$ Partial support by the Swiss National Science Foundation Project 200021-140633/1 and the project RARE -318984 (an FP7 Marie Curie IRSES Fellowship).

${ }^{\ddagger}$ Corresponding author.

can be approximated by a standard Gaussian rv $W$, in such a way that

$$
\lim _{v \rightarrow \infty} \sup _{x \in \mathbb{R}}\left|\mathbb{P}\left\{\zeta_{v}^{*} \leq x\right\}-\mathbb{P}\{W \leq x\}\right|=0 .
$$

Instead of conditioning on $\left\{\zeta_{1}=v\right\}$ one can also condition on the event $\left\{\zeta_{1}>v\right\}$. Again, the same Gaussian approximation of $\zeta_{2}$ given that $\left\{\zeta_{1}>v\right\}$ can be obtained (see Theorem 2.1 below).

The motivation of analyzing the distributional properties of the conditional models stems both from theoryand applied-oriented problems. Commonly in finance and risk management applications there are few observations of risks being large. Therefore, a conditional model, which can be reasonably approximated by some known distribution functions (dfs), is valuable for statistical models; see e.g., $[5,4,14,17,20,26,27,33,6]$ for various results.

Conditional limit results are also crucial for the investigation of the asymptotic behaviour of maximum of random processes and that of maxima of triangular arrays; see e.g., $[1,7,8,28,21,34,2,18]$ and references therein. Other important applications of approximations of the conditional dfs of chi-square risks can be found in [24]. Consider $\zeta_{1, v}$ and $\zeta_{2, v}$ to be realizations of some stationary chi-square process $\{\zeta(t), t \geq 0\}$ at threshold dependent times $t_{1}(v), t_{2}(v)$. In this case we have threshold dependent correlation coefficient $\rho_{v}$ instead of constant $\rho$. In order to get results as those of Berman (see also [1]) we need to assume that $\rho_{v}$ tends to 1 at a certain speed. This case has been considered in the context of maxima of chi-square triangular arrays in Theorem 2.1 in [24], which shows that $\left(\zeta_{2, v}-\zeta_{1, v}\right) \mid\left(\zeta_{1, v}>v\right)$ can be approximated, as $v \rightarrow \infty$, by a Gaussian rv with $\mathrm{df}$ $N(-\lambda, 4 \lambda)$, provided that

$$
\lim _{v \rightarrow \infty} 2 v\left(1-\rho_{v}\right)=\lambda \in(0, \infty) .
$$

Given the importance of approximation of conditional dfs both for constant $\rho$ and for $\rho\left(=\rho_{v}\right)$ that changes with the threshold $v$, in this paper we shall investigate approximations of multivariate conditional perturbed chi-square risks (see Section 2 for the definition) using ideas and techniques from extreme value theory.

Our findings provide a concrete framework for the conditional extreme value model developed in $[27,11]$, and therefore statistical inference can be performed by utilising the 
conditional extreme value methodology therein. Since our approach is asymptotic in nature, distributional assumptions can be dropped. This makes the model more appealing for applications. More precisely, we shall drop any distributional assumption on $X_{i 1}, i \geq 1$. The Gaussianity of the components $W_{i}, i \geq 1$ in (1) seems to be crucial; however there are specific models (see Section 3) where this assumption is relaxed.

In this paper we present three applications: The first application establishes the so-called Berman's sojourn limit theorem and the tail asymptotics of supremum for a class of time-changed stationary chi-square processes. The second application strengthens the convergence in distribution of maxima of chi-square triangular arrays (see [24] and [25]) to convergence of the corresponding probability density functions (pdfs). We conclude Section 4 with the third application concerning extremal behaviour of aggregated log-chi risks.

This contribution is organised as follows: We begin with the description of two main (dependent) perturbed chisquare models for our multivariate framework and then derive conditional limit theorems for the models both with fixed parameters and with parameters that depend on the threshold; see Section 2. Section 3 is devoted to discussions. The aforementioned applications are displayed in Section 4. Proofs of all results are relegated to Section 5 followed by a short Appendix.

\section{MAIN RESULTS}

We first introduce the multidimensional perturbed chisquare random vectors. Let $\left(X_{i 1}, \ldots, X_{i(k+1)}\right), 1 \leq i \leq m$ be $(k+1)$-dimensional random vectors with stochastic representations

$$
\begin{aligned}
& \left(X_{i 1}, \ldots, X_{i(k+1)}\right) \\
& \stackrel{d}{=}\left(X_{i 1}, \rho_{1} X_{i 1}+W_{i 1}, \ldots, \rho_{k} X_{i 1}+W_{i k}\right)
\end{aligned}
$$

$1 \leq i \leq m$, where $\rho_{j} \in \mathbb{R} \backslash\{0\}, 1 \leq j \leq k$, and $\mathcal{W}:=\left\{W_{i j}\right\}_{1 \leq i \leq m, 1 \leq j \leq k}$ is an $m \times k$ matrix of centered (non-standard) Gaussian rvs. Define the $(k+1)$-dimensional perturbed chi-square risk $\zeta:=\left(\zeta_{1}, \ldots, \zeta_{k+1}\right)$ by

$$
\zeta_{1}=\sum_{i=1}^{m} X_{i 1}^{2}, \ldots, \zeta_{k+1}=\sum_{i=1}^{m} X_{i(k+1)}^{2}
$$

In the sequel we shall consider the following framework:

Assumption A: Random vector $\left(X_{11}, \ldots, X_{m 1}\right)$ and the Gaussian random matrix $\mathcal{W}$ are mutually independent. Further, we assume that the rows of $\mathcal{W}$ are independent and have the same df as the centered $k$-dimensional Gaussian random vector $\boldsymbol{W}=\left(W_{1}, \ldots, W_{k}\right)$. Suppose that $\zeta_{1}$ has its support on $[0, \infty)$.

Note that we do not assume $X_{11}, \ldots, X_{m 1}$ to be independent or normally distributed. If they are independent
$N(0,1)$ distributed and for any $1 \leq i \leq m, W_{i j}$ has variance $1-\rho_{j}^{2} \in(0,1)$ for all $1 \leq j \leq k$, then $\zeta$ is the (classical) chi-square risk.

In order to obtain an approximation for the conditional perturbed chi-square risk $\left(\zeta_{2}, \ldots, \zeta_{k+1}\right) \mid\left(\zeta_{1}>v\right)$ we need to impose an asymptotic tail condition on $\zeta_{1}$. We shall assume that $\zeta_{1}$ has df $G$ in the GMDA with positive scaling function $w(\cdot)$, i.e.,

(7) $\lim _{v \rightarrow \infty} \frac{1-G(v+x / w(v))}{1-G(v)}=\exp (-x), \quad x \in \mathbb{R}$.

We refer to $[32,13,15]$ for more details on GMDA. Due to the restrictions imposed by our dependence structure, not every possible scaling function $w(\cdot)$ can be considered. Thus we assume that

$$
\lim _{v \rightarrow \infty}(\sqrt{v} w(v))^{-1}=2 c \in[0, \infty)
$$

Next, we state our first result which shows convergence in distribution of the conditional perturbed chi-square risk. In what follows, the standard notation $\stackrel{d}{\rightarrow}$ and $\stackrel{p}{\rightarrow}$ denote convergence in distribution and convergence in probability, respectively, when the argument tends to infinity.

Theorem 2.1. Let $\boldsymbol{\zeta}:=\left(\zeta_{1}, \ldots, \zeta_{k+1}\right)$ be a perturbed chisquare risk given in (6). Assume that Assumption $A$ is satisfied, and let $\boldsymbol{U}=\left(U_{1}, \ldots, U_{k}\right)$ have the same $d f$ as the centered Gaussian random vector $\boldsymbol{W}$. Then

$$
\begin{array}{r}
\mathbb{P}\left\{\frac{\zeta_{2}-\rho_{1}^{2} v}{2 \rho_{1} \sqrt{v}} \leq x_{1}, \ldots, \frac{\zeta_{k+1}-\rho_{k}^{2} v}{2 \rho_{k} \sqrt{v}} \leq x_{k} \mid \zeta_{1}=v\right\} \\
\rightarrow \mathbb{P}\left\{U_{1} \leq x_{1}, \ldots, U_{k} \leq x_{k}\right\}, \quad v \rightarrow \infty
\end{array}
$$

holds for any $\left(x_{1}, \ldots, x_{k}\right)$ on $\mathbb{R}^{k}$. Further, if $G$ satisfies (7) with some positive scaling function $w(\cdot)$ which satisfies (8), then

$$
\begin{gathered}
\left(w(v)\left(\zeta_{1}-v\right), \frac{\zeta_{2}-\rho_{1}^{2} v}{2 \rho_{1} \sqrt{v}}, \ldots, \frac{\zeta_{k+1}-\rho_{k}^{2} v}{2 \rho_{k} \sqrt{v}}\right) \mid\left(\zeta_{1}>v\right) \\
(10) \stackrel{d}{=}\left(\widetilde{\zeta}_{v}, \widetilde{\boldsymbol{\zeta}}_{v}\right) \stackrel{d}{\rightarrow}\left(E, \rho_{1} c E+U_{1}, \ldots, \rho_{k} c E+U_{k}\right)
\end{gathered}
$$

where $E$ is a unit exponential rv independent of $\boldsymbol{U}$ and $\left(\widetilde{\zeta}_{v}, \widetilde{\zeta}_{v}\right), v>0$ are defined on the same probability space as $\zeta$.

Remark 2.2. In view of Theorem 2.1, Proposition 4.1 in [10] implies that the random vector $\boldsymbol{\zeta}$ has asymptotically independent components, i.e.,

$$
\lim _{v \rightarrow \infty} \mathbb{P}\left\{\zeta_{j}>v \mid \zeta_{i}>v\right\}=0
$$

for any pair $(i, j)$ of different indices; see [25] for a similar result. 
Our second result is concerned with the threshold dependent perturbed $(k+1)$-dimensional chi-square risk $\boldsymbol{\zeta}_{v}:=$ $\left(\zeta_{1, v}, \ldots, \zeta_{k+1, v}\right)$, which is defined similarly as (6) with $\rho_{j, v}, v>0$ instead of $\rho_{j}$ and Gaussian random matrices $\mathcal{W}_{v}, v>0$ instead of $\mathcal{W}$ (note that $\left.\zeta_{1, v}=\zeta_{1}\right)$. For $\rho_{j, v}$ 's we shall impose the following conditions (compare with (4)):

$$
\begin{array}{r}
\lim _{v \rightarrow \infty} 4 v w(v)\left(1-\rho_{j, v}\right)=\lambda_{j} \in[0, \infty), \\
1 \leq j \leq k .
\end{array}
$$

Since $\lim _{v \rightarrow \infty} v w(v)=\infty$, then (12) implies that $\lim _{v \rightarrow \infty} \rho_{j, v}=1$. In the special case that $G$ is a chi-square df, we have $w(v) \equiv 1 / 2$ and thus (12) reduces to (4).

Theorem 2.3. Let $\boldsymbol{\zeta}_{v}:=\left(\zeta_{1, v}, \ldots, \zeta_{k+1, v}\right), v>0$ be a family of threshold dependent perturbed chi-square risks with correlation coefficients $\rho_{j, v} \in \mathbb{R} \backslash\{0\}, 1 \leq j \leq k, v>0$. Denote the first row of $\mathcal{W}_{v}$ by $\boldsymbol{W}_{v}$ and assume that Assumption A holds for every $v>0$. Suppose further that $G$ satisfies (7) with some positive scaling function $w(\cdot)$.

i) Assume that condition (12) is satisfied and

$$
\begin{array}{r}
w(v) \sqrt{v} \boldsymbol{W}_{v} \stackrel{d}{\rightarrow} \boldsymbol{U}, \\
\sqrt{w(v)} \boldsymbol{W}_{v} \stackrel{p}{\rightarrow} \mathbf{0}=(0, \ldots, 0) \in \mathbb{R}^{k}
\end{array}
$$

holds for a random vector $\boldsymbol{U} \in \mathbb{R}^{k}$. Then for any $\left(x_{1}, \ldots, x_{k}\right) \in \mathbb{R}^{k}$

$$
\begin{gathered}
\mathbb{P}\left\{w(v)\left(\zeta_{2, v}-v\right) \leq x_{1}, \ldots, w(v)\left(\zeta_{k+1, v}-v\right) \leq x_{k}\right. \\
\left.\mid \zeta_{1}=v+\frac{x}{w(v)}\right\} \\
\rightarrow \mathbb{P}\left\{2 U_{1}-\frac{\lambda_{1}}{2}+x \leq x_{1}, \ldots, 2 U_{k}-\frac{\lambda_{k}}{2}+x \leq x_{k}\right\}
\end{gathered}
$$

holds locally uniformly for $x \in \mathbb{R}$ as $v \rightarrow \infty$.

ii) If (14) holds locally uniformly for $x \in[0, \infty)$, then

$$
\begin{aligned}
& \left(w(v)\left(\zeta_{1}-v\right), w(v)\left(\zeta_{2, v}-v\right), \ldots, w(v)\left(\zeta_{k+1, v}-v\right)\right) \\
& \quad \mid\left(\zeta_{1}>v\right) \\
& \stackrel{d}{\rightarrow}\left(E, E+2 U_{1}-\frac{\lambda_{1}}{2}, \ldots, E+2 U_{k}-\frac{\lambda_{k}}{2}\right),
\end{aligned}
$$

with $E$ being a unit exponential rv independent of $\boldsymbol{U}$.

An immediate consequence of the above result is the following interesting limit relationship.

Corollary 2.4. Under the assumptions and notation of ii) in Theorem 2.3 we have

$$
\begin{gathered}
\lim _{v \rightarrow \infty} \sup _{\left(x_{1}, \ldots, x_{k}\right) \in \mathbb{R}^{k}} \mid \mathbb{P}\left\{w(v)\left(\zeta_{2, v}-\zeta_{1}\right) \leq x_{1}, \ldots,\right. \\
\left.w(v)\left(\zeta_{k+1, v}-\zeta_{1}\right) \leq x_{k} \mid \zeta_{1}>v\right\}
\end{gathered}
$$

$$
-\mathbb{P}\left\{2 U_{1}-\frac{\lambda_{1}}{2} \leq x_{1}, \ldots, 2 U_{k}-\frac{\lambda_{k}}{2} \leq x_{k}\right\} \mid=0 .
$$

The claim in (16) is of interest for statistical modeling; results in this direction are already available for some other interesting models (see [14]).

Remarks 2.5. a) The relation between (14) and (15) is known from several works of Berman; see e.g., [7] where additional conditions on the scaling function $w(\cdot)$ are imposed.

b) Assume $\boldsymbol{\zeta}_{v}=\left(\zeta_{1, v}, \zeta_{2, v}\right), v>0$ to be a family of 2-dimensional threshold dependent chi-square risks with $\operatorname{Var}\left(W_{i 1, v}\right)=1-\rho_{1, v}^{2} \in(0,1), 1 \leq i \leq m$ and thus $w(x)=1 / 2$. Then from (12) we have that (13) holds with $U_{1}=\sqrt{\lambda_{1}} V_{1} / 2$.

c) The proof of (3) shows that under the assumptions of b), similar convergence as in (14) also holds for the corresponding pdfs.

\section{DISCUSSIONS}

As we can see from the proof of Theorem 2.1 (Eq. (33) therein) the symmetry property of Gaussian rvs plays a crucial role. In this section, we are mainly concerned with two tractable models relaxing the Gaussian assumptions.

First, we consider a bivariate perturbed chi-square risk $\left(\zeta_{1}, \zeta_{2}\right)$ as in (6). We drop the Gaussian assumption on $W_{i 1}, 1 \leq i \leq m$ in $(5)$ and assume that $\left(X_{11}, \ldots, X_{m 1}\right)$ is a random vector with polar representation

$$
\left(X_{11}, \ldots, X_{m 1}\right)=R\left(O_{1}, \ldots, O_{m}\right),
$$

where $R>0$ is a rv with infinite upper endpoint, and $\left(O_{1}, \ldots, O_{m}\right)$ is a random vector which is independent of $R$ and satisfies $\sum_{i=1}^{m} O_{i}^{2}=1$ almost surely. Since

$$
\begin{aligned}
\zeta_{2} & =\rho_{1}^{2} \sum_{i=1}^{m} X_{i 1}^{2}+2 \rho_{1} \sum_{i=1}^{m} X_{i 1} W_{i 1}+\sum_{i=1}^{m} W_{i 1}^{2} \\
& =\rho_{1}^{2} R^{2}+2 R \rho_{1} \sum_{i=1}^{m} O_{i} W_{i 1}+\sum_{i=1}^{m} W_{i 1}^{2}
\end{aligned}
$$

we obtain

$$
\frac{\zeta_{2}-\rho_{1}^{2} v}{2 \rho_{1} \sqrt{v}} \mid\left(\zeta_{1}=v\right) \stackrel{d}{=} \sum_{i=1}^{m} O_{i} W_{i 1}+\frac{\sum_{i=1}^{m} W_{i 1}^{2}}{2 \rho_{1} \sqrt{v}} \stackrel{d}{\rightarrow} \sum_{i=1}^{m} O_{i} W_{i 1}
$$

as $v \rightarrow \infty$. Assume further that $\boldsymbol{O}=\left(O_{1}, \ldots, O_{m}\right)$ is uniformly distributed on the unit sphere of $\mathbb{R}^{m}$. Then (cf. $[8,19])$

$$
\sum_{i=1}^{m} O_{i} W_{i 1} \stackrel{d}{=} O_{1} \sqrt{\sum_{i=1}^{m} W_{i 1}^{2}},
$$

which is in general not Gaussian.

Gaussian approximation of perturbed chi-square risks 365 
Another tractable model is obtained from (6) by restricting the following conditions on the random matrix $\mathcal{W}$. Suppose that each column $\left(W_{1 j}, \ldots, W_{m j}\right), 1 \leq j \leq k$ of the random matrix $\mathcal{W}$ has stochastic representation

$$
\left(W_{1 j}, \ldots, W_{m j}\right)=R_{j} \boldsymbol{O}_{j}=R_{j}\left(O_{1 j}, \cdots, O_{m j}\right),
$$

where $R_{j}$ and $\boldsymbol{O}_{j}$ are independent for any $1 \leq j \leq k$. Here $\boldsymbol{O}_{1}, \ldots, \boldsymbol{O}_{k}$ are independent copies of the random vector $\boldsymbol{O}$ which is uniformly distributed on the unit sphere of $\mathbb{R}^{m}$. It follows that for any $v$ in the support of $\zeta_{1}$

$$
\begin{aligned}
& \left(\zeta_{2}, \ldots, \zeta_{k+1}\right) \mid\left(\zeta_{1}=v\right) \\
& \stackrel{d}{=}\left(\sum_{i=1}^{m}\left(\rho_{1} u_{i}+\widetilde{V}_{1}\right)^{2}, \ldots, \sum_{i=1}^{m}\left(\rho_{k} u_{i}+\widetilde{V}_{k}\right)^{2}\right),
\end{aligned}
$$

where $\widetilde{V}_{j}=R_{j} O_{1 j}, 1 \leq j \leq k$ with $u_{j}, 1 \leq j \leq k$ are such that $\sum_{j=1}^{m} u_{j}^{2}=v$; the proof of (18) is given in Appendix. A direct implication of (18) is that

$$
\begin{aligned}
& \widetilde{\boldsymbol{\zeta}_{v}} \stackrel{d}{=}\left(\frac{\zeta_{2}-\rho_{1}^{2} v}{2 \rho_{1} \sqrt{v}}, \ldots, \frac{\zeta_{k+1}^{2}-\rho_{k}^{2} v}{2 \rho_{k} \sqrt{v}}\right) \mid\left(\zeta_{1}=v\right) \\
& \stackrel{d}{\rightarrow}\left(\widetilde{V}_{1}, \ldots, \widetilde{V}_{k}\right), \quad v \rightarrow \infty
\end{aligned}
$$

Consequently, (9) holds with $\boldsymbol{U} \stackrel{d}{=}\left(\widetilde{V}_{1}, \ldots, \widetilde{V}_{k}\right)$.

Finally, we mention an extension of Theorem 2.3. It is possible therein to drop the assumptions that the rows of the matrix $\mathcal{W}_{v}$ have the same df. To this end, the condition (13) needs to be re-stated, requiring the convergence of $w(v) \sqrt{v} \mathcal{W}_{v}$ to some random matrix $\mathcal{U}$.

\section{APPLICATIONS}

As mentioned in the Introduction, conditional limit results are important in various theoretical and applied models. In this section, we shall present three applications. The first one concerns the derivation of Berman's sojourn limit theorems and the tail asymptotic behaviour of the supremum for certain time-changed stationary chi-square processes. In the second application we shall investigate the maxima of perturbed chi-square triangular arrays establishing both the convergence of the maxima and a density type convergence result. Finally, motivated by the findings of [3], we shall derive the tail asymptotics of aggregated log-chi risks.

\section{Berman's sojourn limit theorem and extremes of time-changed chi-square processes:}

Consider $\left\{X_{i}(t), t \geq 0\right\}, 1 \leq i \leq m$ to be $m$ independent centered stationary Gaussian processes with covariance functions $r_{i}(\cdot), 1 \leq i \leq m$ satisfying

$$
\begin{aligned}
& r_{i}(t)=1-C_{i}|t|^{\alpha}+o\left(|t|^{\alpha}\right), \quad t \rightarrow 0, \\
& r_{i}(t)<1, \quad \forall t>0,
\end{aligned}
$$

with $\alpha \in(0,2]$ and $C_{i}, 1 \leq i \leq m$ given positive constants. Define a time-changed stationary chi-square process $\{\zeta(t), t \geq 0\}$ by

$$
\zeta(t)=\sum_{i=1}^{m} X_{i}^{2}\left(\Theta_{i} t\right), \quad t \geq 0,
$$

where $\boldsymbol{\Theta}=\left(\Theta_{1}, \ldots, \Theta_{m}\right)$ is a random vector with nonnegative and bounded components being independent of the processes $X_{i}, 1 \leq i \leq m$. We remark that time-changed processes are used extensively; see e.g., [12] and references therein. Next, let $\left\{Z_{i}(t), t \geq 0\right\}, 1 \leq i \leq m$ be independent copies of a fractional Brownian motion $\{Z(t), t \geq 0\}$ with Hurst index $\alpha / 2 \in(0,1]$, i.e., a centered Gaussian process with covariance function

$$
\operatorname{Cov}(Z(s), Z(t))=t^{\alpha}+s^{\alpha}-|t-s|^{\alpha}, \quad s, t \geq 0 .
$$

We obtain below a conditional limit result which is crucial for the derivation of Berman's sojourn limit theorems and the tail asymptotic behaviour of the supremum for the time-changed stationary chi-square processes. Since $\zeta(0)$ has a chi-square df, it follows that its df $G$ satisfies (7) with scaling function $w(v) \equiv 1 / 2$. We have, for any $0<t_{1}<t_{2}<\cdots<t_{d}$ and $x>0$ (set $\Delta_{i}\left(t_{j}\right)=$ $X_{i}\left(q(v) t_{j}\right)-r_{i}\left(q(v) t_{j}\right) X_{i}(0), X_{i, v}\left(t_{j}\right):=r_{i}\left(q(v) t_{j}\right) X_{i}(0)$ and $\left.q(v)=v^{-1 / \alpha}\right)$

$$
\begin{aligned}
& \left(w(v)\left(\zeta\left(q(v) t_{1}\right)-v\right), \ldots, w(v)\left(\zeta\left(q(v) t_{d}\right)-v\right)\right) \\
& \mid(\zeta(0)=v+x / w(v)) \\
& \stackrel{d}{=}\left(\frac{1}{2} \sum_{i=1}^{m}\left(\Delta_{i}\left(\Theta_{i} t_{1}\right)\right)^{2}+\sum_{i=1}^{m} \Delta_{i}\left(\Theta_{i} t_{1}\right) X_{i, v}\left(\Theta_{i} t_{1}\right)\right. \\
& +\frac{1}{2}\left(\sum_{i=1}^{m}\left(X_{i, v}\left(\Theta_{i} t_{1}\right)\right)^{2}-\zeta(0)\right)+x, \ldots, \\
& \frac{1}{2} \sum_{i=1}^{m}\left(\Delta_{i}\left(\Theta_{i} t_{d}\right)\right)^{2}+\sum_{i=1}^{m} \Delta_{i}\left(\Theta_{i} t_{d}\right) X_{i, v}\left(\Theta_{i} t_{d}\right) \\
& \left.+\frac{1}{2}\left(\sum_{i=1}^{m}\left(X_{i, v}\left(\Theta_{i} t_{d}\right)\right)^{2}-\zeta(0)\right)+x\right)\left.\right|_{(\zeta(0)=v+2 x) .}
\end{aligned}
$$

By (19) it follows that

$$
\Delta_{i}\left(\Theta_{i} t_{j}\right) \rightarrow 0, \quad r_{i}\left(q(v) \Theta_{i} t_{j}\right) \rightarrow 1
$$

almost surely as $v \rightarrow \infty$ for all $1 \leq i \leq m, 1 \leq j \leq d$. Consequently, the independence of $\Delta_{i}\left(\Theta_{i} t_{j}\right)$ and $\zeta(0)$ implies 


$$
\begin{aligned}
& \left(w(v)\left(\zeta\left(q(v) t_{1}\right)-v\right), \ldots, w(v)\left(\zeta\left(q(v) t_{d}\right)-v\right)\right) \\
& \mid(\zeta(0)=v+x / w(v)) \\
& \stackrel{d}{=}\left(O_{p}(1)+(1+o(1)) \sum_{i=1}^{m} \Delta_{i}\left(\Theta_{i} t_{1}\right) X_{i}(0)\right. \\
& -\frac{1}{2} \sum_{i=1}^{m}\left(1-\left(r_{i}\left(q(v) \Theta_{i} t_{1}\right)\right)^{2}\right)\left(X_{i}(0)\right)^{2}+x, \ldots, \\
& O_{p}(1)+(1+o(1)) \sum_{i=1}^{m} \Delta_{i}\left(\Theta_{i} t_{d}\right) X_{i}(0)-\frac{1}{2} \times \\
& \left.\sum_{i=1}^{m}\left(1-\left(r_{i}\left(q(v) \Theta_{i} t_{d}\right)\right)^{2}\right) X_{i}(0)+x\right) \mid(\zeta(0)=v+2 x) .
\end{aligned}
$$

Furthermore, since $\left(X_{1}(0), \ldots, X_{m}(0)\right)$ is a standard Gaussian random vector, we have the stochastic representation

$$
\left(X_{1}(0), \ldots, X_{m}(0)\right)=R\left(O_{1}, \ldots, O_{m}\right),
$$

where $\left(O_{1}, \ldots, O_{m}\right)$ is a random vector uniformly distributed on the unit sphere of $\mathbb{R}^{m}$ being further independent of $R>0$ which is such that $R^{2}$ has a chi-square df with $m$ degrees of freedom. Hence, in view of the independence between the random variables (or vectors) we conclude that

$$
\begin{aligned}
& \left(w(v)\left(\zeta\left(q(v) t_{1}\right)-v\right), \ldots, w(v)\left(\zeta\left(q(v) t_{d}\right)-v\right)\right) \\
& \mid(\zeta(0)=v+x / w(v)) \\
& \stackrel{d}{=}\left(O_{p}(1)+\sqrt{v+2 x} \sum_{i=1}^{m} \Delta_{i}\left(\Theta_{i} t_{1}\right) O_{i}\right. \\
& -\frac{1}{2}(v+2 x) \sum_{i=1}^{m}\left(1-\left(r_{i}\left(q(v) \Theta_{i} t_{1}\right)\right)^{2}\right) O_{i}^{2}+x, \ldots, \\
& O_{p}(1)+\sqrt{v+2 x} \sum_{i=1}^{m} \Delta_{i}\left(\Theta_{i} t_{d}\right) O_{i}-\frac{1}{2}(v+2 x) \times \\
& \left.\sum_{i=1}^{m}\left(1-\left(r_{i}\left(q(v) \Theta_{i} t_{d}\right)\right)^{2}\right) O_{i}^{2}+x\right) \mid\left(R^{2}=v+2 x\right) \\
& \stackrel{d}{\rightarrow}\left(\sum_{i=1}^{m} Z_{i}\left(C_{i}^{1 / \alpha} \Theta_{i} t_{1}\right) O_{i}-\sum_{i=1}^{m} C_{i} O_{i}^{2} \Theta_{i}^{\alpha} t_{1}^{\alpha}+x, \ldots,\right. \\
& \left.\sum_{i=1}^{m} Z_{i}\left(C_{i}^{1 / \alpha} \Theta_{i} t_{d}\right) O_{i}-\sum_{i=1}^{m} C_{i} O_{i}^{2} \Theta_{i}^{\alpha} t_{d}^{\alpha}+x\right)
\end{aligned}
$$

as $v \rightarrow \infty$, where $Z_{i}, O_{i}, 1 \leq i \leq m, \boldsymbol{\Theta}$ are independent random elements. Consequently, Theorem 2.3 implies the weak convergence of finite dimensional distributions

$$
\begin{aligned}
& \frac{1}{2}(\zeta(q(v) t)-v) \mid(\zeta(0)>v) \stackrel{d}{\rightarrow} \\
& \widetilde{Z(t)}:=\sum_{i=1}^{m} Z_{i}\left(C_{i}^{1 / \alpha} \Theta_{i} t\right) O_{i}-\sum_{i=1}^{m} C_{i} O_{i}^{2} \Theta_{i}^{\alpha} t^{\alpha}+E,
\end{aligned}
$$

where $E$ is a unit exponential $\mathrm{rv}$ which is further independent of all the other random elements. Note that if $C_{i}=C \in$ $(0, \infty), 1 \leq i \leq m$ and $\boldsymbol{\Theta}$ has all components equal to 1 , then

$$
\widetilde{Z(t)} \stackrel{d}{=} Z\left(C^{1 / \alpha} t\right)-C t^{\alpha}+E, \quad t \geq 0
$$

which agrees with the findings of [7].

Define the sojourn time of the process $\zeta$ above a level $v$ in the interval $[0, t]$ by

$$
L_{t}(v)=\int_{0}^{t} \mathbf{1}(\zeta(s)>v) d s, \quad t>0,
$$

where $\mathbf{1}(\cdot)$ is the indicator function. By checking the Assumptions in Theorem 3.1 in [7] (as it was done in Theorem 10.1 therein), we obtain the following Berman's sojourn limit theorem for the time-changed stationary chi-square processes.

Proposition 4.1. Let $\{\zeta(t), t \geq 0\}$ be the time-changed stationary chi-square process with covariance functions satisfying (19), and let $L_{t}(v)$ be defined as in (20). Then, for all $t>0$ small enough

$$
\lim _{v \rightarrow \infty} \int_{x}^{\infty} \frac{\mathbb{P}\left\{v^{1 / \alpha} L_{t}(v)>y\right\}}{v^{1 / \alpha} \boldsymbol{E}\left\{L_{t}(v)\right\}} d y=B(x)
$$

holds at all continuity points $x>0$ of $B(x)=$ $\mathbb{P}\left\{\int_{0}^{\infty} \mathbf{1}(\widetilde{Z(s)}>0) d s>x\right\}$.

Our next result concerns the tail asymptotics of the supremum of $\zeta(t)$ over a fixed interval $[0, T]$. See $[22]$ and the references therein for recent developments in this direction.

Proposition 4.2. Let $\{\zeta(t), t \geq 0\}$ be the time-changed stationary chi-square process with covariance functions satisfying (19). Then, for any $T>0$

$$
\begin{aligned}
\mathbb{P}\left\{\sup _{t \in[0, T]} \zeta(t)>v\right\}= & H_{\alpha}\left[C_{1}, \ldots, C_{m}\right] \frac{2^{1-m / 2} T}{\Gamma(m / 2)} \times \\
& v^{\frac{1}{\alpha}+\frac{m}{2}-1} \exp \left(-\frac{v}{2}\right)(1+o(1)),
\end{aligned}
$$

as $v \rightarrow \infty$, where $\Gamma(\cdot)$ denotes the Euler Gamma function and

$$
H_{\alpha}\left[C_{1}, \ldots, C_{m}\right]=\lim _{a \downarrow 0} \frac{1}{a} \mathbb{P}\left\{\sup _{k \geq 1} \widetilde{Z(a k)} \leq 0\right\} \in(0, \infty) .
$$

Gaussian approximation of perturbed chi-square risks 367 
Maxima of perturbed chi-square triangular arrays:

We write below $H_{\lambda}$ for the bivariate Hüsler-Reiss maxstable df defined as

$$
\begin{aligned}
H_{\lambda}(x, y)=\exp \left(-e^{-x} \Phi\left(\frac{\sqrt{\lambda}}{2}+\frac{y-x}{\sqrt{\lambda}}\right)\right. \\
\left.-e^{-y} \Phi\left(\frac{\sqrt{\lambda}}{2}+\frac{x-y}{\sqrt{\lambda}}\right)\right), \quad x, y \in \mathbb{R},
\end{aligned}
$$

with $\lambda \in(0, \infty)$ the dependence parameter, and $\Phi$ the standard Gaussian df. This distribution appeared initially in [9], and was later studied in [28]. It follows that the pdf $h_{\lambda}$ of $H_{\lambda}$ can be written as

$$
\begin{aligned}
& h_{\lambda}(x, y)=e^{-x} H_{\lambda}(x, y)\left(\frac{1}{\sqrt{\lambda}} \varphi\left(\frac{\sqrt{\lambda}}{2}+\frac{y-x}{\sqrt{\lambda}}\right)\right. \\
& \left.+e^{-y} \Phi\left(\frac{\sqrt{\lambda}}{2}+\frac{y-x}{\sqrt{\lambda}}\right) \Phi\left(\frac{\sqrt{\lambda}}{2}+\frac{x-y}{\sqrt{\lambda}}\right)\right), \quad x, y \in \mathbb{R},
\end{aligned}
$$

with $\varphi$ the pdf of $\Phi$.

Let $\left(X_{i 1}^{(n)}, X_{i 2}^{(n)}\right), 1 \leq i \leq n, n \geq 1$ be a bivariate Gaussian triangular array. Assume that, for any $n \geq 1$, $\left(X_{i 1}^{(n)}, X_{i 2}^{(n)}\right), 1 \leq i \leq n$ are independent bivariate Gaussian random vectors with $N(0,1)$ marginals and correlation $\rho_{n} \in(-1,1) /\{0\}$. The seminal contribution [28] shows that the componentwise maxima of $\left(X_{i 1}^{(n)}, X_{i 2}^{(n)}\right), 1 \leq i \leq n$ is attracted by $H_{\lambda}$ if the Hüsler-Reiss condition

$$
\lim _{n \rightarrow \infty} 4 \ln n\left(1-\rho_{n}\right)=\lambda \in[0, \infty)
$$

holds. Let $H_{n}$ denote the joint df of a bivariate chi-square random vector $\left(\zeta_{1}^{(n)}, \zeta_{2}^{(n)}\right)$ as defined in (2), where in (1) we put $\rho_{n} \in(-1,1) /\{0\}$ instead of $\rho$. In [24] the result of [28] was extended to chi-square case proving that under the condition (24) (set below $t_{n}(x)=a_{n} x+b_{n}$ )

$$
\lim _{n \rightarrow \infty} \sup _{x, y \in \mathbb{R}}\left|\left(H_{n}\left(t_{n}(x), t_{n}(y)\right)\right)^{n}-H_{\lambda}(x, y)\right|=0,
$$

with $a_{n}=2$ and

(26) $b_{n}=2 \ln n+(m-2) \ln (\ln n)-2 \ln \Gamma(m / 2)$.

Later on, in Theorem 2.2 in [25] the same result for a perturbed chi-square vector was obtained, where the Gaussian assumption on $X_{i 1}, 1 \leq i \leq m$ in (2) is removed. Instead therein both marginals $H_{n, j}, i=1,2$ of $H_{n}$ are assumed to be in the GMDA, i.e.,

$$
\lim _{n \rightarrow \infty} \sup _{x \in \mathbb{R}}\left|\left(H_{n, j}\left(t_{n}(x)\right)\right)^{n}-\exp (-\exp (-x))\right|=0
$$

for $j=1,2$, where

$$
a_{n}=1 / w\left(b_{n}\right)=2(1+o(1)), \quad b_{n}=G^{-1}(1-1 / n),
$$

368 K. Debicki, E. Hashorva, and L. Ji with $G=H_{n, 1}$ the df of $\zeta_{1}^{(n)}$, and further the Hüsler-Reiss condition

$$
\lim _{n \rightarrow \infty} 2 \frac{b_{n}}{a_{n}}\left(1-\rho_{n}^{2}\right)=\lambda \in[0, \infty)
$$

holds. Under the conditions (28) and (29), we have by (15) and Remarks 2.5, b) that

$$
\begin{aligned}
& n \mathbb{P}\left\{\zeta_{1}^{(n)}>t_{n}(x), \zeta_{2}^{(n)}>t_{n}(y)\right\} \\
& =\frac{\mathbb{P}\left\{\zeta_{1}^{(n)}>t_{n}(x)\right\}}{\mathbb{P}\left\{\zeta_{1}^{(n)}>b_{n}\right\}} \times \\
& \mathbb{P}\left\{\frac{\zeta_{2}^{(n)}-t_{n}(x)}{a_{n}}>y-x \mid \zeta_{1}^{(n)}>t_{n}(x)\right\}(1+o(1)) \\
& \rightarrow \exp (-x) \mathbb{P}\{\sqrt{\lambda} V-\lambda / 2+E>y-x\}
\end{aligned}
$$

as $n \rightarrow \infty$, where $V$ is an $N(0,1)$ rv independent of the unit exponential rv $E$. This together with (27) implies (25), and thus the claim of Theorem 2.2 in [25] follows. The result stated in (14) can be utilised to extend the convergence of dfs (25) to a convergence of the corresponding pdfs; see e.g., [15] for discussions on the convergence of densities.

Proposition 4.3. Let $\left(\zeta_{1}^{(n)}, \zeta_{2}^{(n)}\right), n \geq 1$ be a family of bivariate chi-square random vectors defined as in (2) with joint $d f H_{n}(x, y)$, where in (1) we put $\rho_{n} \in(-1,1) /\{0\}$ instead of $\rho$. If (29) is satisfied with $a_{n}$ and $b_{n}$ in (26) and $\hat{h}_{n}(x, y)$ is the pdf of $\left(H_{n}\left(t_{n}(x), t_{n}(y)\right)\right)^{n}$ with $t_{n}(x)=$ $a_{n} x+b_{n}$, then

$$
\lim _{n \rightarrow \infty} \hat{h}_{n}(x, y)=h_{\lambda}(x, y)
$$

holds for any $x, y \in \mathbb{R}$.

\section{Aggregation of log-chi risks:}

Let $k \geq 2$, and define $\boldsymbol{\zeta}:=\left(\zeta_{1}, \ldots, \zeta_{k}\right)$ to be a $k$ dimensional chi-square risk with $m$ degrees of freedom defined as in (6) where $W_{i j}$ in (5) has variance $1-\rho_{j}^{2} \in(0,1)$ for any $1 \leq i \leq m, 1 \leq j \leq k-1$. Define further $I_{1}, \ldots, I_{k}$ to be iid Bernoulli rvs with $\mathbb{P}\left\{I_{i}=1\right\}=p=1-\mathbb{P}\left\{I_{i}=-1\right\}$ and $p \in(0,1]$, which are independent of $\zeta$. For any constants $\sigma_{j}>0, \mu_{j} \in \mathbb{R}, 1 \leq j \leq k$, define a $k$-dimensional log-chi risk $\boldsymbol{Z}=\left(Z_{1}, \ldots, Z_{k}\right)$ by

$$
Z_{j}=\exp \left(\sigma_{j} I_{j} \sqrt{\zeta_{j}}+\mu_{j}\right), \quad 1 \leq j \leq k .
$$

The introduction of log-chi risks is motivated by [3] where log-normal risks were considered, which are retrieved when $m=1$ and $p=1 / 2$. As a generalization of the result therein, we obtain the asymptotics of the aggregated log-chi risks.

Proposition 4.4. Let $Z_{1}, \ldots, Z_{k}$ be log-chi risks with $m$ degrees of freedom as above. Let $\tilde{\sigma}:=\sigma_{1} \geq \sigma_{2} \geq \cdots \geq \sigma_{k}>$ 
$0, \tilde{\mu}=\max _{1 \leq j \leq k: \sigma_{j}=\tilde{\sigma}} \mu_{j}$, and $J_{k}=\sharp\left\{1 \leq j \leq k: \sigma_{j}=\right.$ $\left.\tilde{\sigma}, \mu_{j}=\tilde{\mu}\right\}$. Then

$$
\begin{aligned}
& \mathbb{P}\left\{\sum_{j=1}^{k} Z_{j}>u\right\}=\frac{p J_{k}}{2^{m / 2-1} \Gamma(m / 2) \tilde{\sigma}^{m-2}} \times \\
& (\ln u-\tilde{\mu})^{m-2} \exp \left(-\frac{(\ln u-\tilde{\mu})^{2}}{2 \tilde{\sigma}^{2}}\right)(1+o(1))
\end{aligned}
$$

as $u \rightarrow \infty$.

Note in passing that the tail asymptotics of the maximum $\max _{1 \leq j \leq k} Z_{j}$ can be further shown to be tailequivalent with the total risk $\sum_{j=1}^{k} Z_{j}$; see [16] for more examples on this topic.

\section{PROOFS}

Proof of Theorem 2.1. For any $v>0$ we have

$$
\begin{aligned}
& \left(\frac{\zeta_{2}-\rho_{1}^{2} v}{2 \rho_{1} \sqrt{v}}, \ldots, \frac{\zeta_{k+1}-\rho_{k}^{2} v}{2 \rho_{k} \sqrt{v}}\right) \stackrel{d}{=} \\
& \left(\frac{\sum_{i=1}^{m}\left(\rho_{1} X_{i 1}+W_{i 1}\right)^{2}-\rho_{1}^{2} v}{2 \rho_{1} \sqrt{v}}, \ldots,\right. \\
& \left.\frac{\sum_{i=1}^{m}\left(\rho_{k} X_{i 1}+W_{i k}\right)^{2}-\rho_{k}^{2} v}{2 \rho_{k} \sqrt{v}}\right) \stackrel{d}{=} \\
& \left(\frac{2 \rho_{1} \sum_{i=1}^{m} X_{i 1} W_{i 1}+\sum_{i=1}^{m} W_{i 1}^{2}}{2 \rho_{1} \sqrt{v}}, \ldots,\right. \\
& \left.\frac{2 \rho_{k} \sum_{i=1}^{m} X_{i 1} W_{i k}+\sum_{i=1}^{m} W_{i k}^{2}}{2 \rho_{k} \sqrt{v}}\right) .
\end{aligned}
$$

Since further by the independence of $\left(X_{11}, \ldots, X_{m 1}\right)$ and the Gaussian random matrix $\mathcal{W}$ we have

$$
\begin{aligned}
& \left(\sum_{i=1}^{m} X_{i 1} W_{i 1}, \ldots, \sum_{i=1}^{m} X_{i 1} W_{i k}\right) \mid\left(\zeta_{1}=v\right) \stackrel{d}{=} \\
& \left(\sqrt{\sum_{i=1}^{m} X_{i 1}^{2}} W_{1}, \ldots, \sqrt{\sum_{i=1}^{m} X_{i 1}^{2}} W_{k}\right) \mid\left(\zeta_{1}=v\right) \\
& \stackrel{d}{=}\left(\sqrt{v} W_{1}, \ldots, \sqrt{v} W_{k}\right),
\end{aligned}
$$

where (32) can be established by checking the characteristic functions of the rvs on both sides; see e.g., [23]. Thus, the first claim follows immediately by the fact that $\sum_{i=1}^{m} W_{i j}^{2} / \sqrt{v} \stackrel{p}{\rightarrow} 0$ for any $1 \leq j \leq k$.

Next, the assumption that $G$ of $\zeta_{1}$ is in the GMDA implies $\lim _{v \rightarrow \infty} v w(v)=\infty$ and the convergence in distribution

$$
w(v)\left(\zeta_{1}-v\right) \mid\left(\zeta_{1}>v\right) \stackrel{d}{\rightarrow} E, \quad v \rightarrow \infty .
$$

By the above we obtain (set $v_{z}:=v+z / w(v)$ )

$$
\begin{aligned}
\mathbb{P}\left\{\frac{\zeta_{2}-\rho_{1}^{2} v}{2 \rho_{1} \sqrt{v}} \leq x_{1}, \ldots, \frac{\zeta_{k+1}-\rho_{k}^{2} v}{2 \rho_{k} \sqrt{v}} \leq x_{k}\right. \\
\left.\mid \zeta_{1}=v+z / w(v)\right\} \\
=\mathbb{P}\left\{\frac{\zeta_{2}-\rho_{1}^{2} v_{z}+\rho_{1}^{2} z / w(v)}{2 \rho_{1} \sqrt{v_{z}}} \sqrt{v_{z} / v} \leq x_{1}, \ldots,\right. \\
\left.\quad \frac{\zeta_{k+1}-\rho_{k}^{2} v_{z}+\rho_{k}^{2} z / w(v)}{2 \rho_{k} \sqrt{v_{z}}} \sqrt{v_{z} / v} \leq x_{k} \mid \zeta_{1}=v_{z}\right\} \\
\stackrel{(8)}{=} \mathbb{P}\left\{\frac{\zeta_{2}-\rho_{1}^{2} v_{z}}{2 \rho_{1} \sqrt{v_{z}}}+\rho_{1} c z \leq x_{1}, \ldots,\right. \\
\left.\quad \frac{\zeta_{k+1}-\rho_{k}^{2} v_{z}}{2 \rho_{k} \sqrt{v_{z}}}+\rho_{k} c z \leq x_{k} \mid \zeta_{1}=v_{z}\right\}(1+o(1)) \\
\rightarrow \mathbb{P}\left\{W_{1} \leq x_{1}-\rho_{1} c z, \ldots, W_{k} \leq x_{k}-\rho_{k} c z\right\}
\end{aligned}
$$

as $v \rightarrow \infty$, where the convergence holds uniformly with respect to $z \in \mathbb{R}$, meaning that we can substitute $z$ by $z_{v}, v>0$ satisfying $\lim _{v \rightarrow \infty} z_{v}=z \in \mathbb{R}$ in the above. Consequently, in view of Lemma 4.2 in [19], we obtain

$$
\begin{aligned}
& \mathbb{P}\left\{\frac{\zeta_{2}-\rho_{1}^{2} v}{2 \rho_{1} \sqrt{v}} \leq x_{1}, \ldots, \frac{\zeta_{k+1}-\rho_{k}^{2} v}{2 \rho_{k} \sqrt{v}} \leq x_{k} \mid \zeta_{1}>v\right\} \\
& =\int_{v}^{\infty} \mathbb{P}\left\{\frac{\zeta_{2}-\rho_{1}^{2} v}{2 \rho_{1} \sqrt{v}} \leq x_{1}, \ldots, \frac{\zeta_{k+1}-\rho_{k}^{2} v}{2 \rho_{k} \sqrt{v}} \leq x_{k}\right. \\
& \left.\left.\right|_{1}=s\right\} d G(s) /(1-G(v)) \\
& =\int_{0}^{\infty} \mathbb{P}\left\{\frac{\zeta_{2}-\rho_{1}^{2} v}{2 \rho_{1} \sqrt{v}} \leq x_{1}, \ldots, \frac{\zeta_{k+1}-\rho_{k}^{2} v}{2 \rho_{k} \sqrt{v}} \leq x_{k}\right. \\
& \left.\mid \zeta_{1}=v+z / w(v)\right\} d G(v+z / w(v)) /(1-G(v)) \\
& \stackrel{(8)}{\rightarrow} \int_{0}^{\infty} \mathbb{P}\left\{W_{1} \leq x_{1}-\rho_{1} c z, \ldots, W_{k} \leq x_{k}-\rho_{k} c z\right\} \\
& \quad \times \exp (-z) d z, \\
& =\mathbb{P}\left\{U_{1}+\rho_{1} c E \leq x_{1}, \ldots, U_{k}+\rho_{k} c E \leq x_{k}\right\},
\end{aligned}
$$

establishing the proof.

Proof of Theorem 2.3. First note that (12) implies as $v \rightarrow \infty$

$$
w(v)\left(v-\rho_{j, v}^{2} v\right)=2 v w(v)\left(1-\rho_{j, v}\right)(1+o(1))=\lambda_{j} / 2
$$

for $1 \leq j \leq k$. Further, the scaling function $w(\cdot)$ is selfneglecting, i.e.,

$$
\lim _{v \rightarrow \infty} \frac{w(v+x / w(v))}{w(v)}=1, \quad \forall x \in \mathbb{R} .
$$

Therefore, the claim of statement i) follows by the assumption (13) and the convergence in distribution

Gaussian approximation of perturbed chi-square risks 369 


$$
\begin{aligned}
& \left(w(v)\left(\zeta_{2, v}-\rho_{1, v}^{2} v\right), \ldots, w(v)\left(\zeta_{k+1, v}-\rho_{k, v}^{2} v\right)\right) \\
& \mid\left(\zeta_{1}=v\right) \stackrel{d}{\rightarrow}\left(2 U_{1}, \ldots, 2 U_{k}\right),
\end{aligned}
$$

which can be confirmed as in (33), with the aid of the assumption (13). The claim of statement ii) can be established using similar arguments as in (34). This completes the proof.

Proof of Proposition 4.3. Denote by $h_{n}(x, y)$ the pdf of $H_{n}(x, y)$ and write $h_{n, j}, j=1,2$ for its marginal pdfs. Further, write $h_{n}(\cdot \mid x)$ for pdf of $\zeta_{2}^{(n)} \mid \zeta_{1}^{(n)}=x$. By Theorem 2.3 and Remarks $2.5 \mathrm{~b}$ ) we have for any $x, y \in \mathbb{R}$

$\lim _{n \rightarrow \infty} \mathbb{P}\left\{\zeta_{2}^{(n)} \leq t_{n}(y) \mid \zeta_{1}^{(n)}=t_{n}(x)\right\}=\mathbb{P}\left\{V \leq \frac{\sqrt{\lambda}}{2}+\frac{y-x}{\sqrt{\lambda}}\right\}$,

with $V$ an $N(0,1)$ rv. By symmetry

$\lim _{n \rightarrow \infty} \mathbb{P}\left\{\zeta_{1}^{(n)} \leq t_{n}(x) \mid \zeta_{2}^{(n)}=t_{n}(y)\right\}=\mathbb{P}\left\{V \leq \frac{\sqrt{\lambda}}{2}+\frac{x-y}{\sqrt{\lambda}}\right\}$.

Consequently, since $\lim _{n \rightarrow \infty}\left(H_{n}\left(t_{n}(x), t_{n}(y)\right)\right)^{n}=H_{\lambda}(x, y)$

$$
\begin{aligned}
& \hat{h}_{n}(x, y)=a_{n}^{2} n\left(H_{n}\left(t_{n}(x), t_{n}(y)\right)\right)^{n-1} h_{n}\left(t_{n}(y) \mid t_{n}(x)\right) \\
& \times h_{n, 1}\left(t_{n}(x)\right)+a_{n}^{2} n(n-1)\left(H_{n}\left(t_{n}(x), t_{n}(y)\right)\right)^{n-2} \\
& \times h_{n, 1}\left(t_{n}(x)\right) h_{n, 2}\left(t_{n}(y)\right) \mathbb{P}\left\{\zeta_{2}^{(n)} \leq t_{n}(y) \mid \zeta_{1}^{(n)}=t_{n}(x)\right\} \\
& \times \mathbb{P}\left\{\zeta_{1}^{(n)} \leq t_{n}(x) \mid \zeta_{2}^{(n)}=t_{n}(y)\right\} \\
& =(1+o(1)) H_{\lambda}(x, y)\left[a_{n} h_{n}\left(t_{n}(y) \mid t_{n}(x)\right) a_{n} n h_{n, 1}\left(t_{n}(x)\right)\right. \\
& \left.+e^{-x+y} \mathbb{P}\left\{V \leq \frac{\sqrt{\lambda}}{2}+\frac{y-x}{\sqrt{\lambda}}\right\} \mathbb{P}\left\{V \leq \frac{\sqrt{\lambda}}{2}+\frac{x-y}{\sqrt{\lambda}}\right\}\right] .
\end{aligned}
$$

Since $G$ is a chi-square df we have $\lim _{n \rightarrow \infty} n a_{n} h_{n 1}\left(t_{n}(x)\right)=$ $\exp (-x)$. Further, in the light of Remarks $2.5 \mathrm{c}$ ) we obtain that

$$
\lim _{n \rightarrow \infty} a_{n} h_{n}\left(t_{n}(y) \mid t_{n}(x)\right)=g(y \mid x),
$$

with $g(\cdot \mid x)$ the pdf of $\sqrt{\lambda} V-\lambda / 2+x$, implying thus

$$
\begin{aligned}
& \lim _{n \rightarrow \infty} \hat{h}_{n}(x, y)=e^{-x} H_{\lambda}(x, y)\left[\frac{1}{\sqrt{\lambda}} \varphi\left(\frac{\sqrt{\lambda}}{2}+\frac{y-x}{\sqrt{\lambda}}\right)\right. \\
& \left.+e^{-y} \Phi\left(\frac{\sqrt{\lambda}}{2}+\frac{y-x}{\sqrt{\lambda}}\right) \Phi\left(\frac{\sqrt{\lambda}}{2}+\frac{x-y}{\sqrt{\lambda}}\right)\right] \\
& =h_{\lambda}(x, y),
\end{aligned}
$$

hence the proof is complete.

Proof of Proposition 4.4. The proof is based on Theorem 4.2 in [30]. Let $\tilde{Z}=\exp \left(\tilde{\sigma} I_{1} \sqrt{\zeta_{1}}+\tilde{\mu}\right)$. Since $\zeta_{1}$ is a chi-square df with $m$ degrees of freedom, it follows that (e.g., $[23])$

$$
\begin{aligned}
& \mathbb{P}\{\tilde{Z}>u\}=\frac{p}{2^{m / 2-1} \Gamma(m / 2) \tilde{\sigma}^{m-2}}(\ln u-\tilde{\mu})^{m-2} \\
& \quad \times \exp \left(-\frac{(\ln u-\tilde{\mu})^{2}}{2 \tilde{\sigma}^{2}}\right)(1+o(1))
\end{aligned}
$$

as $u \rightarrow \infty$, implying that $\tilde{Z}$ has $\mathrm{df}$ in the GMDA with scaling function $w(x)=(\ln x) /\left(\tilde{\sigma}^{2} x\right)$. Since further $\lim _{x \rightarrow \infty} w(x)=$ 0 , in view of Theorem 4.2 in [30] we conclude the claim by checking Assumptions 2.3-2.5 therein. In our setup it suffices to show these assumptions for $k=2$. For the simplicity of presentation, we assume further that $\sigma_{1}=\sigma_{2}=1, p=1$ and $\mu_{1}=\mu_{2}=0$. For any $a>0$ we have

$$
\begin{aligned}
& \mathbb{P}\left\{w(u) Z_{2}>a \mid Z_{1}>u\right\}=\mathbb{P}\left\{Z_{2}>a u / \ln u \mid Z_{1}>u\right\} \\
& \stackrel{v=(\ln u)^{2}}{=} \mathbb{P}\left\{\zeta_{2}>(\ln a+\sqrt{v}-\ln \sqrt{v})^{2} \mid \zeta_{1}>v\right\} \\
& =\mathbb{P}\left\{\frac{\zeta_{2}-\rho_{1}^{2} v}{\sqrt{v}}>\sqrt{v}\left[(1+(\ln a-\ln \sqrt{v}) / \sqrt{v})^{2}-\rho_{1}^{2}\right]\right. \\
& \left.\quad \mid \zeta_{1}>v\right\} \rightarrow 0
\end{aligned}
$$

as $u \rightarrow \infty$, where the last convergence follows from Theorem 2.1 and the fact that $\rho_{1}^{2}<1$, hence Assumption 2.3 and Assumption 2.4 (by symmetry) in [30] hold. The Assumption 2.5 in [30] follows if we show that

$$
\frac{\mathbb{P}\left\{\min \left(Z_{1}, Z_{2}\right)>u / \ln u\right\}}{\mathbb{P}\left\{Z_{1}>u\right\}}=\frac{\mathbb{P}\left\{\min \left(\zeta_{1}, \zeta_{2}\right)>v^{*}\right\}}{\mathbb{P}\left\{\zeta_{1}>(\ln u)^{2}\right\}} \rightarrow 0
$$

as $u \rightarrow \infty$, where $v^{*}=(\ln u-\ln \ln u)^{2}=(\ln u)^{2}(1+o(1))$. By the definition of $\left(\zeta_{1}, \zeta_{2}\right)$ we have the stochastic representation

$$
\zeta_{1}+\zeta_{2} \stackrel{d}{=}\left(1+\rho_{1}\right) \sum_{i=1}^{m} W_{i}^{2}+\left(1-\rho_{1}\right) \sum_{i=m+1}^{2 m} W_{i}^{2},
$$

where $W_{i}, 1 \leq i \leq 2 m$ are iid $N(0,1)$ rvs. Let $\boldsymbol{X}=$ $\left(X_{11}, X_{12}, X_{21}, X_{22}, \cdots, X_{m 1}, X_{m 2}\right)$. The last formula follows by the fact that the covariance matrix $\Sigma$ of $\boldsymbol{X}$ can be written as $\Sigma=\boldsymbol{A} \operatorname{diag}\left(\lambda_{1}, \cdots, \lambda_{2 m}\right) \boldsymbol{A}^{\top}$, where $\lambda_{1}=\cdots=$ $\lambda_{m}=1-\rho_{1}, \lambda_{m+1}=\cdots=\lambda_{2 m}=1+\rho_{1}$, and $\boldsymbol{A} \in \mathbb{R}^{m \times m}$ is some orthogonal matrix. Without loss of generality we may assume that $\rho_{1}>0$. Let $c:=2 /\left(1+\rho_{1}\right)>1$. We have

$$
\begin{aligned}
& \mathbb{P}\left\{\min \left(\zeta_{1}, \zeta_{2}\right)>u\right\} \leq \mathbb{P}\left\{\zeta_{1}+\zeta_{2}>c\left(1+\rho_{1}\right) u\right\} \\
& =\left(1+1 / \rho_{1}\right)^{m / 2} \frac{2^{1-m} c^{m / 2-1}}{\Gamma(m / 2)} u^{m / 2-1} \\
& \quad \times \exp \left(-\frac{c u}{2}\right)(1+o(1))
\end{aligned}
$$

as $u \rightarrow \infty$; see e.g., [23]. Consequently,

$$
\lim _{u \rightarrow \infty} \frac{\mathbb{P}\left\{\min \left(Z_{1}, Z_{2}\right)>u / \ln u\right\}}{\mathbb{P}\left\{Z_{1}>u\right\}}
$$

370 K. Débicki, E. Hashorva, and L. Ji 


$$
\leq \lim _{v \rightarrow \infty} \frac{\mathbb{P}\left\{\zeta_{1}+\zeta_{2}>c\left(1+\rho_{1}\right) v^{*}\right\}}{\mathbb{P}\left\{\zeta_{1}>v^{*}(1+o(1))\right\}}=0
$$

and thus the proof is complete.

\section{ACKNOWLEDGEMENTS}

We are thankful to the referees for several suggestions which improved our manuscript.

\section{APPENDIX}

In this section we first present the proof of (18) and then give a direct proof of (3).

For notational simplicity we consider only the case $k=2$. We have, for any $v$ in the support of $\zeta_{1}$

$$
\begin{aligned}
& \left(\zeta_{2}, \zeta_{3}\right) \mid\left(\zeta_{1}=v\right) \\
& \stackrel{d}{=}\left(\sum_{i=1}^{m}\left(\rho_{1} X_{i 1}+W_{i 1}\right)^{2}, \sum_{i=1}^{m}\left(\rho_{2} X_{i 1}+W_{i 2}\right)^{2}\right) \mid\left(\zeta_{1}=v\right) \\
& \stackrel{d}{=}\left(\rho_{1}^{2} v+2 \rho_{1} \sum_{i=1}^{m} X_{i 1} W_{i 1}+\sum_{i=1}^{m} W_{i 1}^{2},\right. \\
& \left.\rho_{2}^{2} v+2 \rho_{2} \sum_{i=1}^{m} X_{i 1} W_{i 2}+\sum_{i=1}^{m} W_{i 2}^{2}\right) \mid\left(\zeta_{1}=v\right) .
\end{aligned}
$$

where $($ set $N=m+1)$

$$
U_{m}=\sum_{i=1}^{N}\left(X_{i 1}-\overline{X_{N 1}}\right)^{2}, \quad V_{m}=\sum_{i=1}^{N}\left(X_{i 2}-\overline{X_{N 2}}\right)^{2}
$$

and

$$
\overline{X_{N 1}}:=\sum_{i=1}^{N} \frac{X_{i 1}}{N}, \quad \overline{X_{N 2}}:=\sum_{i=1}^{N} \frac{X_{i 2}}{N},
$$

which follow from the facts that $\left(U_{m}, V_{m}\right)$ is independent of $\left(\overline{X_{N 1}}, \overline{X_{N 2}}\right)$, and $\left(\overline{X_{N 1}} \sqrt{N}, \overline{X_{N 2}} \sqrt{N}\right)$ has the same $\mathrm{df}$ as $\left(X_{11}, X_{12}\right)$. From equation (3) in [31] we have the following expression for the pdf $h_{m}$ of $\left(U_{m}, V_{m}\right)$ :

$$
\begin{aligned}
& h_{m}(u, v)=\frac{(u v)^{m / 2-1}}{2^{m}(\Gamma(m / 2))^{2}\left(\rho_{*}^{2}\right)^{m / 2}} \exp \left(-\frac{u+v}{2 \rho_{*}^{2}}\right) \\
& \times{ }_{0} F_{1}\left(; \frac{m}{2} ; \frac{\rho^{2} u v}{\left(2 \rho_{*}^{2}\right)^{2}}\right), \quad \forall u, v \in(0, \infty),
\end{aligned}
$$

where $\rho_{*}:=\sqrt{1-\rho^{2}}$ and ${ }_{0} F_{1}(; a ; x)=\sum_{n=0}^{\infty} \frac{\Gamma(a+n)}{\Gamma(a)} \frac{x^{n}}{n !}$. By (35) the pdf $g_{m}(x \mid v), x \in \mathbb{R}$ of the conditional rv

$$
Z_{v}^{*}=\frac{\zeta_{2}-\rho^{2} v}{\rho_{*} \sqrt{v}} \mid\left(\zeta_{1}=v\right)
$$

is given by (set $x_{\rho}:=\tilde{\rho} x \sqrt{v}+\rho^{2} v$ where $\left.\tilde{\rho}:=1-\rho^{2}\right)$

$$
\begin{aligned}
& g_{m}(x \mid v)=\frac{\tilde{\rho} \sqrt{v}}{\Gamma(m / 2)(2 \tilde{\rho})^{m / 2}} x_{\rho}^{(m-2) / 2} \exp \left(-\frac{x \sqrt{v}}{2}\right) \\
& \times \exp \left(-\frac{\rho^{2} v}{\tilde{\rho}}\right){ }_{0} F_{1}\left(; \frac{m}{2} ; \frac{\rho^{2} v x_{\rho}}{(2 \tilde{\rho})^{2}}\right) .
\end{aligned}
$$

Utilising the well-known asymptotic expansion

$$
\begin{aligned}
& \left.\rho_{2}^{2} v+2 \rho_{2} R_{2} O_{12} \sqrt{\sum_{i=1}^{m} X_{i 1}^{2}}+R_{2}^{2}\right) \mid\left(\sum_{i=1}^{m} X_{i 1}^{2}=v\right) \\
\stackrel{d}{=} & \left(\rho_{1}^{2} v+2 \rho_{1} R_{1} O_{11} \sqrt{v}+R_{1}^{2},\right. \\
& \left.\rho_{2}^{2} v+2 \rho_{2} R_{2} O_{21} \sqrt{v}+R_{2}^{2}\right) \\
\stackrel{d}{=} & \left(\sum_{i=1}^{m}\left(\rho_{1} u_{i}+W_{i 1}\right)^{2}, \sum_{i=1}^{m}\left(\rho_{2} u_{i}+W_{i 2}\right)^{2}\right)
\end{aligned}
$$

for any $u_{i}, i \leq m$ such that $\sum_{i=1}^{m} u_{i}^{2}=v$, where in the first equality in distribution we used the same technique as in (17). Hence the claim of (18) follows.

Next, we show the proof of (3). In view of [29] (see also [31]), we have the stochastic representation

$$
\left(\zeta_{1}, \zeta_{2}\right) \stackrel{d}{=}\left(U_{m}, V_{m}\right)
$$

$$
\begin{aligned}
& { }_{0} F_{1}(; m ; z)=\frac{\Gamma(m)}{2 \sqrt{\pi}} z^{1 / 4-m / 2} \exp (2 \sqrt{z})(1+O(1 / \sqrt{z})) \\
& \text { as } z \rightarrow \infty, \text { we can further write as } v \rightarrow \infty \\
& \quad g_{m}(x \mid v)=\frac{\tilde{\rho} \sqrt{v}}{\Gamma(m / 2)(2 \tilde{\rho})^{m / 2}} x_{\rho}^{(m-2) / 2} \exp \left(-\frac{x \sqrt{v}}{2}\right) \\
& \quad \times \exp \left(-\frac{\rho^{2} v}{2 \tilde{\rho}}\right) \frac{\Gamma(m / 2)}{2 \sqrt{\pi}}\left(\frac{\rho^{2} v x_{\rho}}{(2 \tilde{\rho})^{2}}\right)^{(1-m) / 4} \\
& \quad \times \exp \left(\left(\frac{\rho^{2} v x_{\rho}}{\tilde{\rho}^{2}}\right)^{1 / 2}\right)(1+o(1)) \\
& =\frac{1}{2 \sqrt{\pi}} \frac{\sqrt{v} \tilde{\rho}}{\sqrt{2 \tilde{\rho} \rho v}} \exp \left(-\frac{x \sqrt{v}}{2}\right) \exp \left(-\frac{\rho^{2} v}{\tilde{\rho}}\right) \\
& \quad \times \exp \left(\frac{\rho^{2} v}{\tilde{\rho}}\left(1+\frac{\tilde{\rho} x}{\rho^{2} \sqrt{v}}\right)^{1 / 2}\right)(1+o(1))
\end{aligned}
$$

Gaussian approximation of perturbed chi-square risks 371 


$$
\begin{aligned}
& =\frac{1}{2 \sqrt{\pi}} \frac{\sqrt{\tilde{\rho}}}{\sqrt{2 \rho}} \exp \left(-\frac{x \sqrt{v}}{2}\right) \exp \left(-\frac{\rho^{2} v}{\tilde{\rho}}\right) \\
& \times \exp \left(\frac{\rho^{2} v}{\tilde{\rho}}\left(1+\frac{\tilde{\rho} x}{2 \rho^{2} \sqrt{v}}-\frac{1}{8} \frac{\tilde{\rho}^{2} x^{2}}{\rho^{4} v}+o(v)\right)\right)(1+o(1)) \\
& =\frac{1}{\sqrt{2 \pi}} \frac{\sqrt{\tilde{\rho}}}{2 \sqrt{\rho}} \exp \left(-\frac{\tilde{\rho} x^{2}}{8 \rho^{2}}+o(v)\right)(1+o(1)) \\
& =\frac{1}{\sqrt{2 \pi}} \frac{\sqrt{\tilde{\rho}}}{2 \sqrt{\rho}} \exp \left(-\frac{\tilde{\rho} x^{2}}{8 \rho^{2}}\right)(1+o(1)) .
\end{aligned}
$$

Consequently, for any $x \in \mathbb{R}$

$$
\begin{aligned}
& \mathbb{P}\left\{\frac{\zeta_{2}-\rho^{2} v}{2 \rho \sqrt{1-\rho^{2}} \sqrt{v}} \leq x \mid \zeta_{1}=v\right\} \\
& =\mathbb{P}\left\{Z_{v}^{*} \leq \frac{2 \rho x}{\sqrt{1-\rho^{2}}}\right\} \\
& \rightarrow \mathbb{P}\left\{W_{1} \leq x\right\}, \quad v \rightarrow \infty .
\end{aligned}
$$

The uniform convergence (in $x$ ) of the last formula follows since both functions on the right and left hand sides are continuous, bounded and increasing.

\section{Received 14 September 2013}

\section{REFERENCES}

[1] Albin J. M. P. (1990). On extremal theory for stationary processes. Ann. Probab. 18 92-128. MR1043939

[2] Albin J. M. P. and Choi H. (2010). A new proof of an old result by Pickands. Electron. Commun. Probab. 15 339-345. MR2685014

[3] Asmussen S. and Rojas-Nandayapa L. (2008). Asymptotics of sums of lognormal random variables with Gaussian copula. Statist. Probab. Lett. 78 2709-2714. MR2465111

[4] Balkema A. A. and Embrechts P. (2007). High risk scenarios and extremes: A geometric approach. Zurich Lectures in Advanced Mathematics. European Mathematical Society (EMS), Zürich. MR2372552

[5] Barbe P. (2003). Approximation of integrals over asymptotic sets with applications to probability and statistics. arXiv:math/0312132.

[6] Barbe P. and Seifert M. I. (2013). A conditional limit theorem for a bivariate representation of a univariate random variable and conditional extreme values. arXiv:1311.0540.

[7] Berman S. M. (1982). Sojourns and extremes of stationary processes. Ann. Probab. 10 1-46. MR0637375

[8] Berman S. M. (1992). Sojourns and Extremes of Stochastic Processes. Wadsworth \& Brooks/Cole, Pacific Grove, CA.

[9] Brown B. M. and Resnick S. I. (1977). Extreme values of independent stochastic processes. J. Appl. Probab. 14 732-739. MR0517438

[10] Das B. and Resnick S. I. (2011). Conditioning on an extreme component: Model consistency with regular variation on cones. Bernoulli 17 226-252. MR2797990

[11] Das B. and Resnick S. I. (2011). Detecting a conditional extreme value model. Extremes 14 29-61. MR2775870
[12] Dẹbicki K., Hashorva E. and Ji L. (2014). Tail asymptotics of supremum of certain Gaussian processes over threshold dependent random intervals. Extremes, in press.

[13] Embrechts P., Klüppelberg C. and Mikosch T. (1997). Modelling Extremal Events for Insurance and Finance. SpringerVerlag, Berlin.

[14] Engelke S., Malinowski A., Kabluchko Z. and Schlather M. (2014). Estimation of Hüsler-Reiss distributions and BrownResnick processes. J.R. Statist. Soc. B, in press.

[15] Falk M., HÜsler J. and Reiss R. D. (2010). Laws of Small Numbers: Extremes and Rare Events. In DMV Seminar, volume 23, page 3rd edn. Birkhäuser, Basel.

[16] Foss S., Korshunov D. and Zachary S. (2011). An Introduction to Heavy-tailed and Subexponential Distributions. SpringerVerlag, New York. MR2810144

[17] Fougk̀nes A. L. and Soulier P. (2012). Estimation of conditional laws given an extreme component. Extremes 15 1-34. MR2891307

[18] French J. P. and Davis R. A. (2013). The asymptotic distribution of the maxima of a Gaussian random field on a lattice. Extremes 16 1-26. MR3020174

[19] Hashorva E. (2007). Conditional limiting distribution of type III elliptical random vectors. J. Multivariate Anal. 98 282-294. MR2301753

[20] Hashorva E. (2012). Exact tail asymptotics in bivariate scale mixture models. Extremes 15 109-128. MR2891312

[21] Hashorva E. (2013). Minima and maxima of elliptical triangular arrays and spherical processes. Bernoulli 19 886-904. MR3079299

[22] Hashorva E., Ji L. and Piterbarg V. I. (2013). On the supremum of $\gamma$-reflected processes with fractional Brownian motion as input. Stochastic Process. Appl. 123 4111-4127. MR3091101

[23] Hashorva E., Ji L. and Tan Z. (2012). On the infinite sums of deflated Gaussian products. Electron. Commun. Probab. 17 1-8. MR2955496

[24] Hashorva E., Kabluchko Z. and Wübker A. (2012). Extremes of independent chi-square random vectors. Extremes 15 35-42. MR2891308

[25] Hashorva E., Nadaraja S. and Pogani T. K. (2013). Extremes of perturbed bivariate rayleigh risks. REVSTAT - Statistical Journal 1645-6726.

[26] Heffernan J. E. and Resnick S. I. (2007). Limit laws for random vectors with an extreme component. The Annals of Applied Probability 17 537-571. MR2308335

[27] Heffernan J. E. and Tawn J. A. (2004). A conditional approach for multivariate extreme values (with discussion). J.R. Statist. Soc. B 66 497-546. MR2088289

[28] Hüsler J. and Reiss R. D. (1989). Maxima of normal random vectors: Between independence and complete dependence. Statist. Probab. Lett. 7 283-286. MR0980699

[29] Joarder A. H., Laradji A. and Omar M. H. (2012). On some characteristics of bivariate chi-square distribution. Statistics $\mathbf{4 6}$ 577-586. MR2974639

[30] Mitra A. and Resnick S. I. (2009). Aggregation of rapidly varying risks and asymptotic independence. Adv. in Appl. Probab. 41 797-828. MR2571317

[31] Nadarajah S. (2010). Simple expressions for a bivariate chisquare distribution. Statistics 44 189-201. MR2674417

[32] RESNICK S. I. (1987). Extreme values, regular variation, and point processes. Springer-Verlag, New York. MR0900810

[33] Resnick S. I. and Zeber D. (2013). Markov kernels and the conditional extreme value model. arXiv:1210.3060.

[34] Turkman K. F., Turkman M. A. A. and Pereira J. M. (2010). Asymptotic models and inference for extremes of spatio-temporal data. Extremes 13 375-397. MR2733939 
Krzysztof Dębicki

Mathematical Institute

University of Wroctaw

pl. Grunwaldzki 2/4, 50-384 Wroctaw

Poland

E-mail address: Krzysztof.Debicki@math.uni.wroc.pl

Enkelejd Hashorva

Department of Actuarial Science

University of Lausanne

UNIL-Dorigny 1015 Lausanne

Switzerland

E-mail address: Enkelejd.Hashorva@unil.ch
Lanpeng Ji

Department of Actuarial Science

University of Lausanne

UNIL-Dorigny 1015 Lausanne

Switzerland

E-mail address: Lanpeng.Ji@unil.ch 